\title{
On the prediction of the auroral westward electrojet index
}

\author{
O. A. Amariutei ${ }^{1}$ and N. Yu. Ganushkina ${ }^{1,2}$ \\ ${ }^{1}$ Finnish Meteorological Institute, Helsinki, Finland \\ ${ }^{2}$ University of Michigan, Ann Arbor, USA \\ Correspondence to: O. A. Amariutei (olga.amariutei@fmi.fi)
}

Received: 1 November 2011 - Revised: 19 March 2012 - Accepted: 4 May 2012 - Published: 14 May 2012

\begin{abstract}
An ARMAX based model, to forecast the evolution of the of AL index, is developed. The model has been trained and validated using neural networks with the half wave rectifier $\left(V B_{\mathrm{s}}\right)$ as input. It is shown that the model posses a good, reliable forecasting ability, including periods of intense geomagnetic activity. Prediction efficiency of the model is discussed in the context of 1 min resolution output smoothed over $7 \mathrm{~min}$.
\end{abstract}

Keywords. Magnetospheric physics (Storms and substorms)

\section{Introduction}

Many contemporary technological systems are susceptible to the adverse effects caused by space weather disturbances, so the forecasting of space weather dynamics has become one of the most important applications of space physics. A number of indices were proposed to qualify the intensity of the disturbances in the magnetosphere. Auroral electrojet indices are obtained from a number (usually greater than 10) of stations distributed in local time in the latitude region that is typical of the Northern Hemisphere auroral zone (Davis and Sugiura, 1966). For each of the stations the north-south magnetic perturbation $\mathrm{H}$ is recorded as a function of universal time. A superposition of these data from all the stations enables a lower bound or maximum negative excursion of the $\mathrm{H}$ component to be determined; this is called the $\mathrm{AL}$ index. Similarly, an upper bound or maximum positive excursion in $\mathrm{H}$ is determined; this is called the AU index. The difference between these two indices, AU-AL, is called the AE index. Notice that negative $\mathrm{H}$ perturbations occur when stations are under an westward-flowing current. Thus the indices AU and AL give some measure of the individual strengths of eastward and westward electrojets, while AE provides a measure of the overall horizontal current strength. During auroral substorms excursions in the auroral indices from a nominal daily baseline, with durations of tens of minutes to several hours, are seen. Therefore, times of sharp drops in, for example, $\mathrm{Al}$ index are considered as onset times for substorms. The sharp increase of energetic (a few tens to hundreds of $\mathrm{keV}$ ) particle flux in the near-Earth tail is one of the most important manifestations of the substorm expansion phase. The electric field behaviour is important in understanding how the energetic particle injections are formed and how the particles are accelerated. Observations show that substorm-associated electric fields usually display a very complicated behaviour. The enhanced electric fields are impulsive with amplitudes of up to $20 \mathrm{mV} \mathrm{m}^{-1}$, which is more than three times the largest convection electric field that coincides with the braking of the fast flows, and it is correlated with the magnetic field dipolarization in the inner central plasma sheet (Tu et al., 2000, and references therein). Several models have been proposed to explain particle injections (Li et al., 1998; Sarris et al., 2002). The models are built on the idea that a perturbation farther out in the magnetotail propagates inward, probably in the form of bursty bulk flows, and produces dipolarization and dispersionless injections. So far these models give a good agreement with the observed dispersionless electron injections at geostationary orbit.

The relative importance of the large-scale convection electric field and the substorm-associated electric fields in the energization and transport of particles from the plasma sheet into the ring current region is still an open question. Ganushkina et al. (2005, 2006), using Inner Magnetosphere Particle Transport and Acceleration Model (IMPTAM) (Ganushkina et al., 2001, 2005, 2006), were able to reproduce the observed amount of ring current protons with energies of $80 \mathrm{keV}$ during a storm recovery phase. In addition to the large-scale fields, transient fields associated with 
the dipolarization process in the magnetotail during substorm onset were included for this modeling. These fields were modeled as earthward propagating electromagnetic pulses of localised radial and longitudinal extent (Li et al., 1998; Sarris et al., 2002).

The times when the electromagnetic pulses were launched, were the substorm onset times (Ganushkina et al., 2001, 2005, 2006). The times of sharp drops in the AL index were used for the onset times and the amplitudes of the pulses were scaled proportionally with the magnitudes of the peaks in the AL index. Therefore, for the modeling of the processes of particle transport and acceleration from the plasma sheet to the inner magnetosphere regions, the information given by the AL index is of a great importance. The auroral indices, $\mathrm{AL}, \mathrm{AU}, \mathrm{AE}$ and $\mathrm{AO}$, being a relative measure of the global auroral electrojet activity, give rise to notable challenges in prediction. Forecasting the auroral westward region electrojet currents, which are quantified by the AL index driven by solely the solar wind and interplanetary magnetic field (IMF), is of great importance and has stimulated numerous studies.

In the present paper, the proposed AL forecasting model has been developed and optimised as an addition to the IMPTAM model due to the necessity of including the electromagnetic pulses that will be launched at times and scaled with the relative amplitudes given by the AL forecast. In order to provide a near real time prediction, we have built a model based on previous achievements in nonlinear system identification and foregoing advances in modeling auroral indices. Low-dimensional system identification has been suggested as an appropriate technique in the search of a model for the magnetospheric dynamics (Sharma, 1995; Roberts et al., 1991; Boynton et al., 2011a; Vassiliadis and Klimas, 1995). Nonlinear models for the AL prediction, driven by the solar wind and IMF parameters, have been pioneered by Bargatze et al. (1985) and further developed by Vassiliadis (1994), Price et al. (1994) and Vassiliadis et al. (1995). Neural networks have been introduced into prediction of auroral indices along with nonlinear models (ARMA and MA filters) by Hernandez et al. (1993) and later on by Gleisner and Lundstedt (1997) and Weigel et al. (1999). Following the steps of Hernandez et al. (1993), our model uses neural nets to construct nonlinear models using Autoregressive Moving Average with eXogenous input(s) (ARMAX). The data and the challenges of the data sets in correlation with the model are discussed in Sect. 2, while results and conclusions are presented in Sects. 3 and 4, respectively.

\section{Data and model}

The input data of our model are the half wave rectifier (Burton et al., 1975) $V B_{\mathrm{s}}$, where $V$ is the solar wind velocity and $B_{\mathrm{S}}$ is defined as the $\mathrm{z}$-component of the interplanetary magnetic field (IMF), when southward- orientated or zero

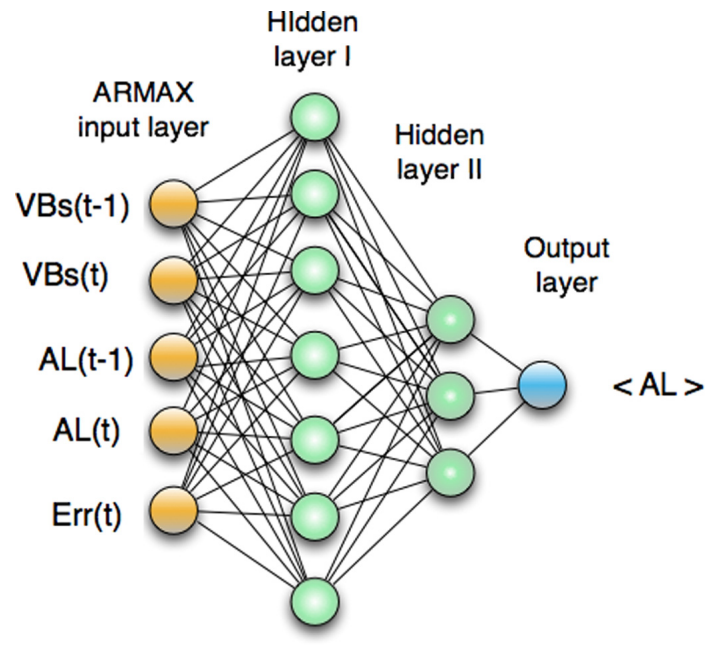

Fig. 1. A schematic representation of the ARMAX - neural network.

otherwise (in GSM coordinates). The output is the AL index data. We used $1 \mathrm{~min}$ resolution data for solar wind and IMF parameters observed at ACE spacecraft, propagated to the sub solar point of the magnetopause by omniweb (http: //omniweb.gsfc.nasa.gov/form/omni_min.html). The AL index data were obtained from the World Data Center for Geomagnetism, Kyoto (http://wdc.kugi.kyoto-u.ac.jp/). We used 1 January 1998 to 31 December 1998 for training of the model and 1 January 1999 to 31 May 1999 for validation. There are three main technical issues that come into consideration: data gaps, the ballistic propagation of the data from in situ measurements to the magnetopause and the resolution of the data. The comprehensive analysis of nonlinear systems always require continuous data sets. In the data set considered, we have found only a few data gaps, so the overall duration of the gaps does not have great impact on the results. We apply a simple method, based on a recurrent linear interpolation algorithm, to replace the missing data points with the most fitted value given by the nearest neighbour to the gap. The second problem is related to the propagation methods used for shifting the data measured at L1 to the magnetopause. While this is an issue that is beyond the scope of our presented work, it will be discussed in Sect. 3 in terms of the model's performance and capabilities. In the present study, in order to identify drivers for the IMPTAM model, the emphasis falls on an accurate forecasting of the times more than the amplitudes of the modelled data series, therefore the 1-min resolution data has been smoothed with a moving average over an interval of $7 \mathrm{~min}$, similar to $\mathrm{Li}$ et al. (2007) (10 or 15 min averaged data) and Weigel et al. (1999).

ARMAX is often used to model systems of short time scales. ARMAX modeling technique is a variation of the general AutoRegressive Moving Average (ARMA) model. 

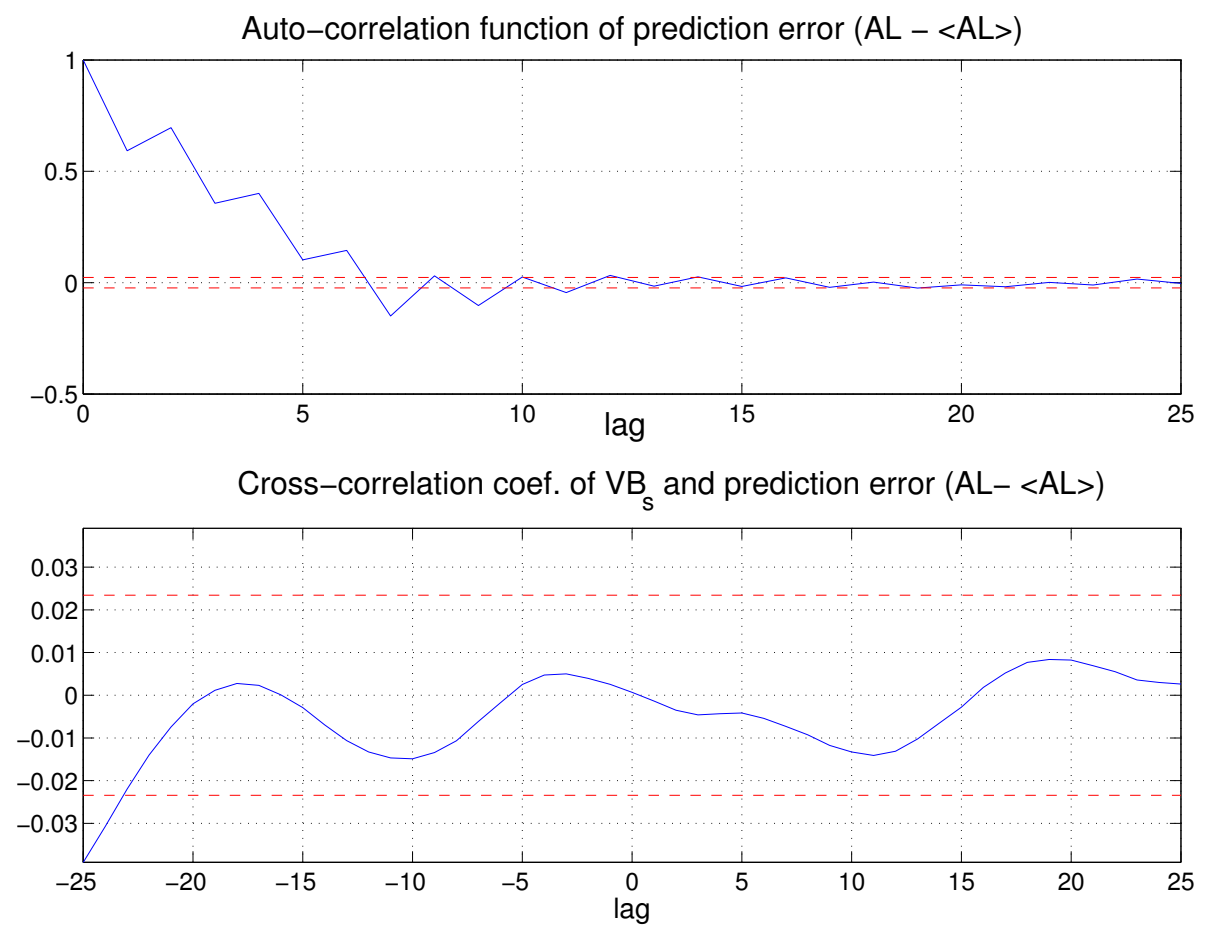

Fig. 2. Top panel: the autocorrelation function of the prediction error. Bottom panel: the cross correlation between the input $\left(V B_{\mathrm{S}}\right)$ and the prediction error.

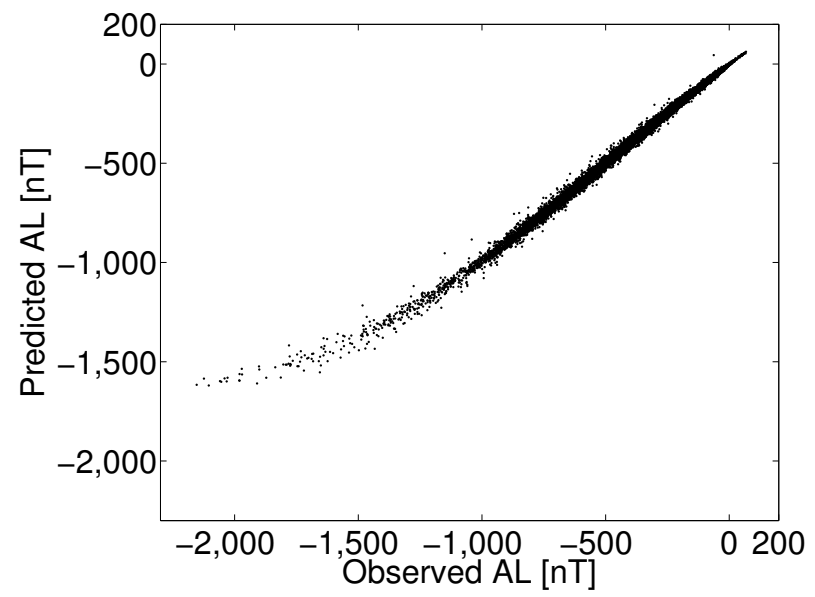

Fig. 3. Observed AL vs. predicted AL, using data from validation testbed (January-May 1999).

The general ARMA equation is given by

$y(n)=-\sum_{k=1}^{p} a_{k} y(n-k)+\sum_{k=0}^{q} b_{k} x(n-k)$,

where $y(n)$ is the output of the system, $x(n)$ is the input of the system, $b_{k}$ and $a_{k}$ are coefficients of the $k$-th polynomial shift operator. The incorporation of an external input, called exogenous term, to the systems results would give the formulation for the ARMAX model (Eq. 2) as

$$
y(n)=-\sum_{k=1}^{p} a_{k} y(n-k)+\sum_{k=0}^{q} b_{k} x(n-k)+\sum_{k=1}^{r} c_{k} u(n-k) .
$$

The extra term $u$ and its coefficient $c_{k}$ is the exogenous input sequence, and this term represents the past prediction errors. At the initial run of the model, this is assumed to be zero, and during the training epochs it will get the value of the difference between the desirable output and the predicted output (Billings and Voon, 1986; Boynton et al., 2011b). Different methods exist to determine the coefficients of the ARMA/ARMAX model, such as Least square methods, Shank method, Pade approximation or covariance/autocorrelation methods. In the present work, we propose another method, which exploits the main ability of the neural network. Neural networks are commonly used when the exact nature of the relationship between inputs and output is unknown. The most important feature of the neural networks is their capability to learn the relationship between input and output through training. We propose a setup based on an ARMAX model (Eq. 2), for which a neural network method is used for the determination of the coefficients. The feed forward neural network consist of one input layer (with various number of inputs), one or several hidden layers (with various numbers of units) and the output layer which can also have several outputs. The processing units also called 


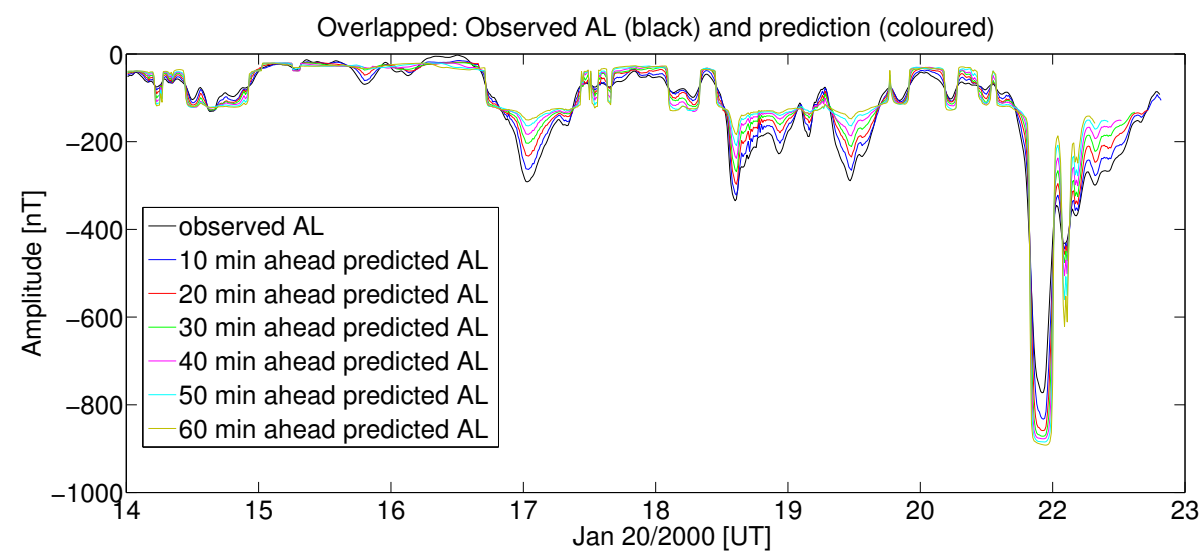

Fig. 4. Model performance at various prediction steps, ranging from 10 to $60 \mathrm{~min}$ ahed. The figure depicts an event that took place on 20 January 2000.

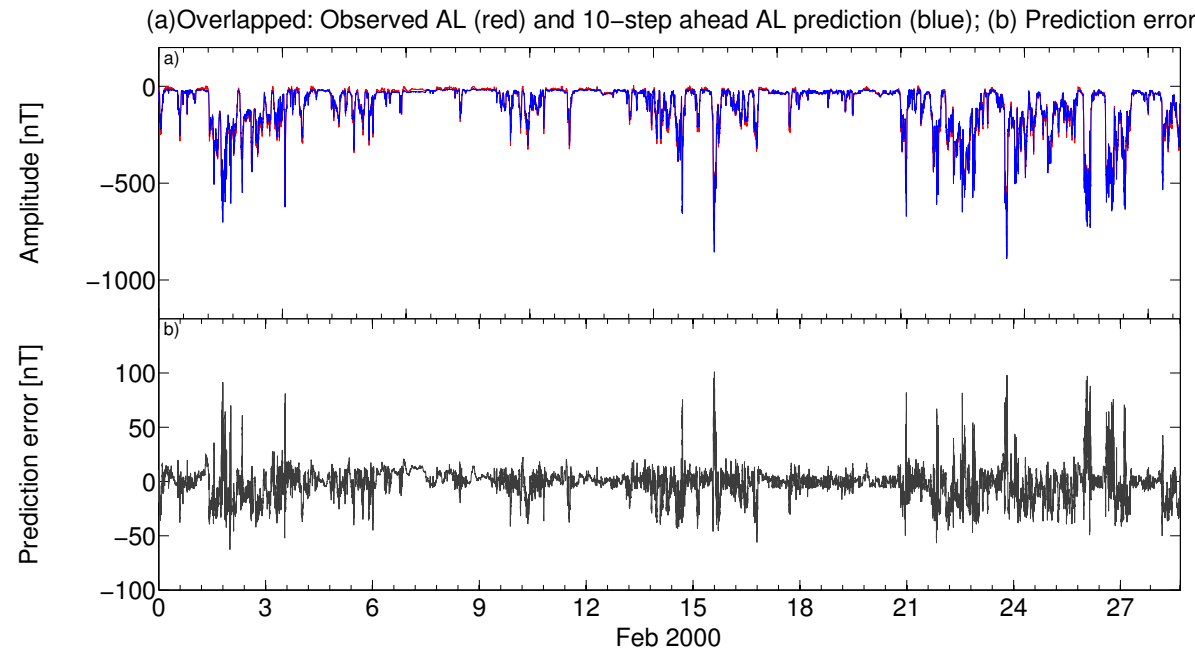

Fig. 5. (a) AL observation (red) and predicted AL (blue) - 10 step ahead prediction; (b) prediction error. The panel shows one month of data (February 2000) at 1 min resolution.

nodes, are interconnected with neighbouring nodes from adjacent layers. The parameters associated with these connections are called weights. The hidden layers, in our case two, are weighted by two matrixes W1 (input to hidden) and W2 (hidden to output). The first hidden layer consists of seven units, activated by a hyperbolic tangential function, which ensures a higher coefficient variation for the activation values of the hidden unit. The second layer has a linear activation function for each of the two units (Hernandez et al., 1993), as displayed in Fig. 1. A reliable method for determining the number of the hidden layers and units is still under dispute. While few general rules are nowadays accepted (Chester, 1990), the extended verification methods are beyond the scope of this work (Lawrence et al., 1996). The neural network structure was chosen to suit the nonlinearity of the system and the number of the hidden units was chosen in accordance with the number of inputs/outputs of the network (Tetko et al., 1995). The network is trained to estimate its response by adjusting the set of the weights (W1 and W2), in accordance with the "learning rule". The learning law is given by back propagation of the error between the desired output, and the systems output and it has been implemented using Levenberg-Marquardt (Kisi, 2004; Catalao et al., 2007) method. Figure 1 shows a schematic representation of the model.

\section{AL prediction results}

The network training has been done for 1 min resolution measurements of the solar wind and IMF parameters and AL index for the whole year 1998. The sum of squared deviations of actual values from predicted values was $\mathrm{SSE}=18$ $\left(\mathrm{nT}^{2}\right)$ and the prediction efficiency was $\mathrm{PE}=0.4$, where $\mathrm{PE}$ is defined as the proportion between the mean squared residual 


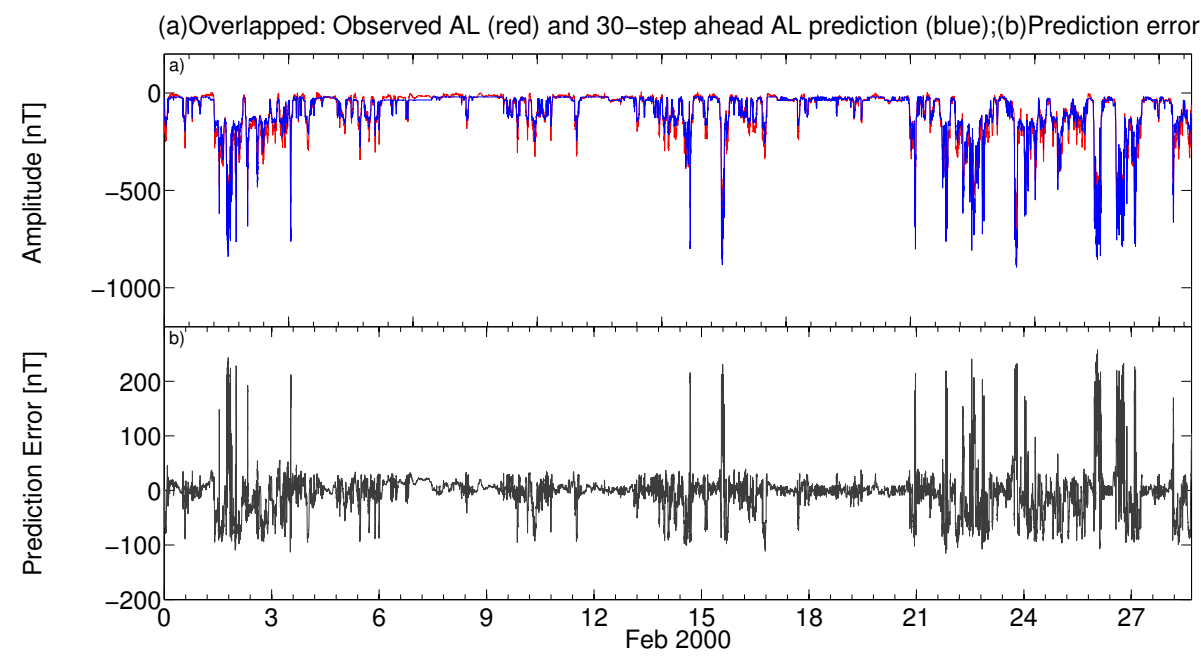

Fig. 6. (a) AL observation (red) and predicted AL (blue) - 30 step ahead prediction; (b) prediction error. The panel shows one month of data (February 2000) at 1 min resolution.

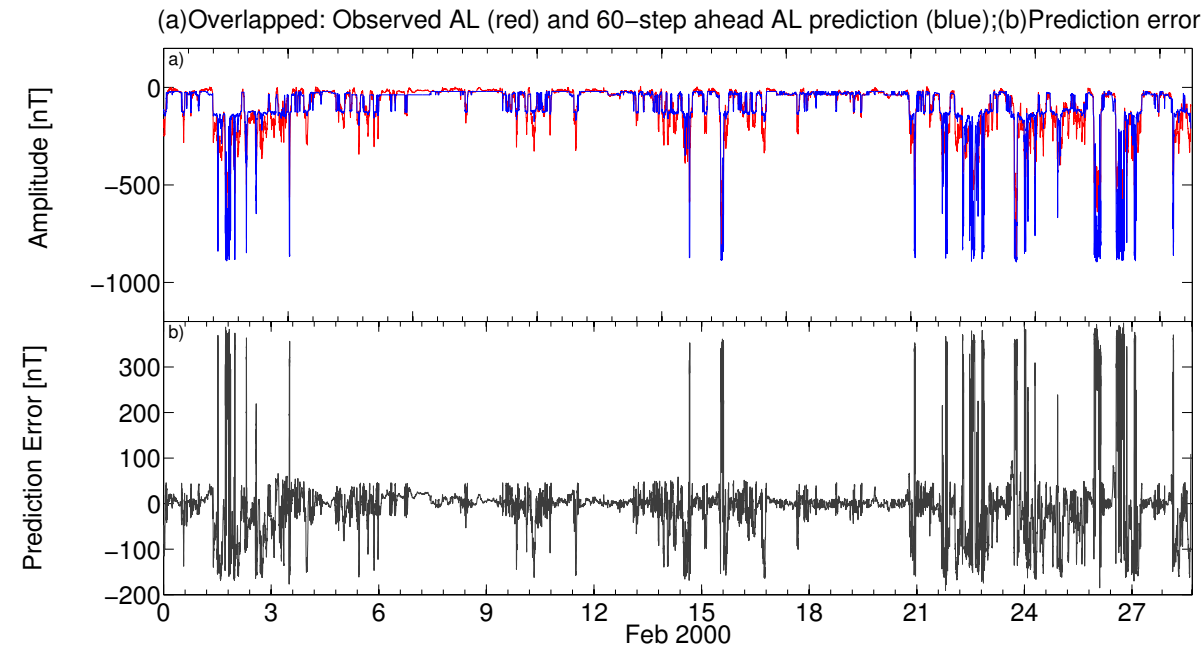

Fig. 7. (a) AL observation (red) and predicted AL (blue) - 60 step ahead prediction; (b) prediction error. The panel shows one month of data (February 2000) at 1 min resolution.

and the variance of the coefficients and SSE is the sum of squared errors. Once the model has been trained, we choose a different data set, from January to end of May 1999, for the validation of the model. Figure 2 shows the model error after validation for one step ahead prediction. The top panel shows the $95 \%$ confidence interval for the autocorrelation function of the prediction error, while the bottom panel shows the cross-correlation of the input with the prediction error. Both the autocorrelation and cross-correlation fall within the confidence range. For the autocorrelation, the reason behind the 7 lags before falling into the confidence range, can be explained in the context of the data being smoothed over 7 points. Figure 3 shows the scatter plot between the observed $\mathrm{AL}$ index and predicted $\mathrm{AL}$ index for the validation set. It is noticeable that the points align along the $45^{\circ}$ line, which means a very good correlation. The scatters on the sides of the $45^{\circ}$ line, depict either the over- or under-estimation of the observed values by the prediction model. As expected, during intense magnetospheric activity, the model overestimated the magnitude of the sub-storm. This shows that the neural network structure is relevant to the system and that we do not need to increase the order of the model (Billings and Voon, 1986; Billings and Zhu, 1989).

The prediction of the AL index for a chosen event can be seen in Fig. 4 for 10 to 60 steps ahead. The chosen event took place on 20 January 2000 and illustrates the precision of the algorithm in determining the exact times in the occurrence of the event. The model capability over a longer period of time is illustrated in 3 different figures: for $10 \mathrm{~min}$ ahead Fig. 5, 30 min ahead Fig. 6 and 60 min ahead Fig. 7. 
The top panel (a) in every figure shows the observed vs. predicted AL values and the bottom panel (b) shows the prediction error, defined as the difference between the measured and the predicted AL. The panels represent a month of data (February 2000) at 1 min resolution. Figure 5 shows a prediction with a small overestimation of AL amplitudes ranging from 10 to $70 \mathrm{nT}$, with a prediction efficiency $\mathrm{PE}=0.98$ and a normalised squared mean error NRMSE $=0.113$, computed as the square root of the proportion between the sum of the squares of the residuals and the variance of the data. The 30 step ahead forecast (Fig. 6) accurately predicts the timing of the substorm onsets with small overestimations of the magnitude during large amplitudes of AL and small underestimations during quiet times. The model overestimation during the intensive activity is of the order of approximatively $200 \mathrm{nT}$ NRMSE $=0.33$ and a $\mathrm{PE}=0.89$. The $60 \mathrm{~min}$ forecast displays a better result than expected (Fig. 7) with a NRMSE $=0.61$ and a $\mathrm{PE}=0.63$, following the same pattern as the $30 \mathrm{~min}$ ahead prediction, underestimating lower values and overestimating during substorms. However, it should be noticed that the prediction is following the shape of the observed AL data. As mentioned before, ACE data, propagated to the magnetopause is used as input. At the beginning of February 2000, ACE spacecraft was orbiting around the Sun-Earth L1 libration point, at $238 R_{\mathrm{E}}$ sunward of the Earth, spinning further away towards $242 R_{\mathrm{E}}$ at the end of the month. The relative distance to magnetopause is ranging from 230 to $232 R_{\mathrm{E}}$, and the velocity of the bulk plasma flow spans from $311 \mathrm{~km} \mathrm{~s}^{-1}$ to $989 \mathrm{~km} \mathrm{~s}^{-1}$, which implies that time of arrival to the magnetopause would be between over $30 \mathrm{~min}$ to less than $80 \mathrm{~min}$. Making predictions for 60 steps ahead, in this context, is an ambitious task, as the model would predict solely on what it has learned and cannot rely much on solar wind inputs. At small IMF velocities, the parameters for the half wave rectifier $\left(V B_{\mathrm{S}}\right)$ do not arrive in time at the magnetopause, forcing the neural-ARMAX model to estimate the output relying only on past learned behaviour.

\section{Conclusions}

We have presented a new model for prediction of the AL index, based on a combination of neural networks with an ARMAX model. Statistical evaluation of the model shows that it is reliable and it is fit to be used as an addition to the Inner Magnetosphere Particle Transport and Acceleration Model (IMPTAM). The existing prediction models for the AL index provide either a parametric model (Li et al., 2007) or models dependent on a prior classification of the data (Hernandez et al., 1993). The former provides a batch prediction with the disadvantage of completely missing the onsets of some of the events, while the latter would give great prediction but only on a carefully selected set of input data, which would make it impossible to use with live data. The neural network and AR-
MAX model that we have proposed, has a number of advantages, not only for our future use: the algorithm is not computationally expensive, it is able to account for nonlinearities in the input-output relationship and it provides a stable and reliable prediction. Furthermore, the algorithm gives acceptable results even in extreme conditions, when input data (solar wind parameters) are scarcely available, as in the case of $60 \mathrm{~min}$ ahead prediction.

Acknowledgements. 1 min resolution data for solar wind and IMF parameters observed at ACE spacecraft, were obtained from OMNIWeb (http://omniweb.gsfc.nasa.gov/form/omni_min.html). The AL index data were obtained from the World Data Center for Geomagnetism, Kyoto (http://wdc.kugi.kyoto-u.ac.jp/). The research leading to these results has received funding from the European Union Seventh Framework Programme (FP7/2007-2013) under grant agreement No. 262468, and is also supported in part by the Finnish Meteorological Institute, Helsinki, Finland. N. Ganushkina gratefully acknowledges NASA and NSF for sponsoring this research through various research grants.

Topical Editor R. Nakamura thanks three anonymous referees for their help in evaluating this paper.

\section{References}

Bargatze, L. F., Baker, D. N., McPherron, R. L., and Hones, E. W., J.: Magnetospheric Impulse Response for Many Levels of Geomagnetic Activity, J. Geophys. Res., 90, 6387-6394, 1985.

Billings, S. A. and Voon, W. S. F.: Correlation based model validity tests for non-linear models, Int. Control J., 44, 235-244, 1986.

Billings, S. A. and Zhu, Q. M.: Nonlinear model validation using correlation tests, Int. Control J., 62, 749-766, 1989.

Boynton, R. J., Balikhin, M. A., Billings, S. A., Sharma, A. S., and Amariutei, O. A.: Data derived NARMAX Dst model, Ann. Geophys., 29, 965-971, doi:10.5194/angeo-29-965-2011, 2011a.

Boynton, R. J., Balikhin, M. A., Billings, S. A., Wei, H. L., and Ganushkina, N.: Using the NARMAX OLS-ERR algorithm to obtain the most influential coupling functions that affect the evolution of the magnetosphere, J. Geophys. Res., 116, A05218, doi:10.1029/2010JA015505, 2011b.

Burton, R. K., McPherron, R. L., and Russell, C. T.: The Terrestrial Magnetosphere: A Half-Wave Rectifier of the Interplanetary Electric Field, Science, 189, 717-718, 1975.

Catalao, J. P. S., Mariano, S. J. P. S., Mendes, V. M. F., and Ferreira, L. A. F. M.: Short-term electricity prices forecasting in a competitive market: A neural network approach, Electric Power Systems Research, 77, 1297-1304, doi:10.1016/j.epsr.2006.09.022, 2007.

Chester, D.: Why two layers are better than one, vol. 1, IJCNN-90WASH-DC, Lawrence Erlbaum, 1990.

Davis, T. N. and Sugiura, M.: Auroral Electrojet Activity Index AE and Its Universal Time Variations, J. Geophys. Res., 71, 785801, 1966.

Ganushkina, N. Y., Pulkkinen, T. I., Bashkirov, V. F., Baker, D. N., and Li, X.: Formation of intense nose structures, Geophys. Res. Lett., 28, 491-494, 2001.

Ganushkina, N. Yu., Pulkkinen, T. I., and Fritz, T.: Role of substorm-associated impulsive electric fields in the ring cur- 
rent development during storms, Ann. Geophys., 23, 579-591, doi:10.5194/angeo-23-579-2005, 2005.

Ganushkina, N. Y., Pulkkinen, T. I., Milillo, A., and Liemohn, M.: Evolution of the proton ring current energy distribution during 21-25 April 2001 storms, J. Geophys. Res., 111, A11S08, doi:10.1029/2006JA011609, 2006.

Gleisner, H. and Lundstedt, H.: Response of the auroral electrojets to the solar wind modeled with neural networks, J. Geophys. Res., 102, 14269-14278, 1997.

Hernandez, J. V., Tajima, T., and Horton, W.: Neural net forecasting for geomagnetic activity, Geophys. Res. Lett., 20, 2707-2710, 1993.

Kisi, O.: Multi-layer perceptrons with Levenberg-Marquardt training algorithm for suspended sediment concentration prediction and estimation, Hydrol. Sci. J.-journal Des Sciences Hydrologiques, 49, 1025-1040, doi:10.1623/hysj.49.6.1025.55720, 2004.

Lawrence, S., Giles, C., and Tsoi, A.: What size neural network gives optimal generalization? Convergence properties of backpropagation, Technical Report UMIACS-TR-96-22 and CS-TR3617, Institute for Advanced Computer Studies, University of Maryland, College Park, MD 20742, 1996.

Li, X., Baker, D. N., Temerin, M., Reeves, G. D., and Belian, R. D.: Simulation of dispersionless injections and drift echoes of energetic electrons associated with substorms, Geophys. Res. Lett., 25, 3763-3766, 1998.

Li, X., Oh, K. S., and Temerin, M.: Prediction of the AL index using solar wind parameters, J. Geophys. Res., 112, A06224, doi:10.1029/2006JA011918, 2007.

Price, C. P., Prichard, D., and Bischoff, J. E.: Nonlinear Input/Output Analysis of the Auroral Electrojet Index, J. Geophys. Res., 99, 13227-13238, 1994.
Roberts, D. A., Baker, D. N., Klimas, A. J., and Bargatze, L. F.: Indications of low dimensionality in magnetospheric dynamics, Geophys. Res. Lett., 18, 151-154, 1991.

Sarris, T. E., Li, X., Tsaggas, N., and Paschalidis, N.: Modeling energetic particle injections in dynamic pulse fields with varying propagation speeds, J. Geophys. Res., 107, 1033, doi:10.1029/2001JA900166, 2002.

Sharma, A.: Assessing the magnetosphere's nonlinear behavior: Its dimension is low, its predictability, high (95RG00495), Tech. rep., 1995.

Tetko, I. V., Livingstone, D. J., and Luik, A. I.: Neural network studies. 1. Comparison of overfitting and overtraining, J. Chem. Inf. Comput. Sci., 35, 826-833, 1995.

Tu, J.-N., Tsuruda, K., Hayakawa, H., Matsuoka, A., Mukai, T., Nagano, I., and Yagitani, S.: Statistical nature of impulsive electric fields associated with fast ion flow in the near-Earth plasma sheet, J. Geophys. Res., 105, 18901-18907, 2000.

Vassiliadis, D.: The input-state space approach to the prediction of auroral geomagnetic activity from solar wind variables, in: Applications of Artificial Intelligence in Solar Terrestrial Physics, edited by: Joselyn, J. H. L. and Trolinger, J., vol. NOAA/SEC, Boulder, CO, pp. 145-151, 1994.

Vassiliadis, D. and Klimas, A. J.: On the Uniqueness of Linear Moving-Average Filters for the Solar Wind-Auroral Geomagnetic Activity Coupling, J. Geophys. Res., 100, 5637-5641, 1995.

Vassiliadis, D., Klimas, A. J., Baker, D. N., and Roberts, D. A.: A Description of the Solar Wind-Magnetosphere Coupling Based on Nonlinear Filters, J. Geophys. Res., 100, 3495-3512, 1995.

Weigel, R. S., Horton, W., Tajima, T., and Detman, T.: Forecasting auroral electrojet activity from solar wind input with neural networks, Geophys. Res. Lett., 26, 1353-1356, 1999. 